\title{
Tissue Engineering - The Gateway to Regenerative Medicine
}

\author{
Epifania Bono, Stephanie H. Mathes, Nicola Franscini, and Ursula Graf-Hausner*
}

\begin{abstract}
Tissue Engineering as an emerging biotechnology sector aims at the in vitro regeneration of diseased tissues and promises to profoundly change medical practice, offering the possibility of regenerating tissues and organs instead of just repairing them (regenerative medicine). Improved healing processes and a higher quality of life are the expected results. This article gives an overview of different technologies for regenerative medicine and presents results of our own current applied research and development. A recent project was successfully closed with the development of a natural biomaterial for soft tissue oral defects. The establishment of an in vitro bioreactor system enabled us to simulate the mechanical and biological environment in a healing wound and to investigate the suitability of different implant materials for the oral tissue regeneration. Moreover, focusing the attention on an alternative method for the intervertebral disc (IVD) regeneration, we established a new tissue engineered approach, based on the three-dimensional (3D) culture of autologous human IVD cells into a polyurethane (PU)-fibrin composite. IVD cells were able to proliferate and, thanks to the 3D conditions, to differentiate expressing the typical native tissue markers. The development of an automated platform was the goal of an additional project, to standardize the cell culture technology, increase the bio-safety and reduce the production costs, moving tissue engineering nearer to clinical application.
\end{abstract}

Keywords: Automation · Biomaterials · Disc regeneration · Soft tissue $\cdot$ Tissue regeneration

\section{Introduction}

Age today no longer has the meaning it used to have. Because people live longer, the medical and scientific community is therefore expected to provide for good health and solutions to various degenerative ailments. This is where regenerative medicine steps in and offers innovative treatments and cures. Tissue engineering provides the opportunity to regenerate impaired tissues (and organs, as a future perspective) using in vitro techniques or a combination of in vitro and in vivo processes. Although this technology field was first defined by Langer and Vacanti ${ }^{11]}$ as early as 1993, the clinical application and commercialization of biological products

${ }^{\star}$ Correspondence: Prof. Dr. U. Graf-Hausner Tel.: + 410589345518 Fax: + 410589345659

E-mail: ursula.graf@zhaw.ch

Institute of Chemistry and Biological Chemistry

Zurich University of Applied Sciences

Campus Reidbach

Einsiedlerstrasse 31

$\mathrm{CH}-8820$ Waedenswil are still in their initial phase. The interdisciplinary field of tissue engineering applies the principles of engineering and life sciences toward the development of biological substitutes that restore, maintain or improve tissue or organ function. [1] Several products, currently on the market, are suitable for skin substitution (e.g. Dermagraft ${ }^{\circledR}$, Advanced BioHealing, United States; Apligraf ${ }^{\circledR}$, Organogenesis Inc., United States; Epicel ${ }^{\circledR}$, Genzyme Biosurgery, United Kingdom), knee cartilage repair (autologous chondrocyte transplantation, ATC by co.don AG, Germany; Carticel $^{\circledR}$, Genzyme Biosurgery, United Kingdom) and a few for bone repair. Most of the products are relatively simple and consist of only one cell type. Others that are more advanced are under development or in clinical trials, such as bioartificial organs like urinary bladder (e.g. Neo-Bladder Augment ${ }^{\mathrm{TM}}$, Tengion, United States) $)^{[2]}$ and liver, ${ }^{[3]}$ or for cardiovascular diseases. ${ }^{[4]}$ Even if several scientific and technical hurdles still need to be overcome (e.g. vascularisation, controlled three-dimensional structure, coordinated action of different cell types), the use of stem cells, the improvement of the bio- and implant materials as well as the controlled release of bioactive signaling factors enhance the potential of tissue engineering technologies. Commercial expectations are high regarding the potential markets which tissue-engineered products could cover. Estimations are in the range of 4-400 billion Euro per year worldwide. ${ }^{[5]}$

\section{Two Main Strategies: in vitro and in vivo}

Cells, scaffolds and signaling factors represent the core of all tissue-engineering strategies. In addition the producing technologies, like bioreactor systems, applying perfusion and biomechanical stimulation are crucial. The basic idea is to recreate a structure able to respond to biological and mechanical signals, and to interact with the surrounding host tissue after the implantation. Biomaterial scaffolds provide a critical means of controlling engineered tissue architecture and mechanical properties, functioning as a carrier for cells and biomolecules such as growth factors. The procedure of the in vitro strategy is a new functional tissue based on a biodegradable and resorbable scaffold, which is colonized in vitro with autologous cells of the patient and later implanted. As the cells grow and produce extracellular matrix (ECM) (Fig. 1), the new tissue substitutes the scaffold that is gradually degraded, resorbed or metabolized. Biological and technical experience have developed rapidly over the last 10 years, but the legislation and regulations for the use of living cells for transplantation are restrictive and hurdles in the approval process for biological products are huge.

The scaffold can also be implanted into the patient without cells. The acellular biomaterial with bioactive behavior will enhance the repair of the tissue, e.g. bone. The regenerative process will be performed by the body itself in vivo. This strategy is 


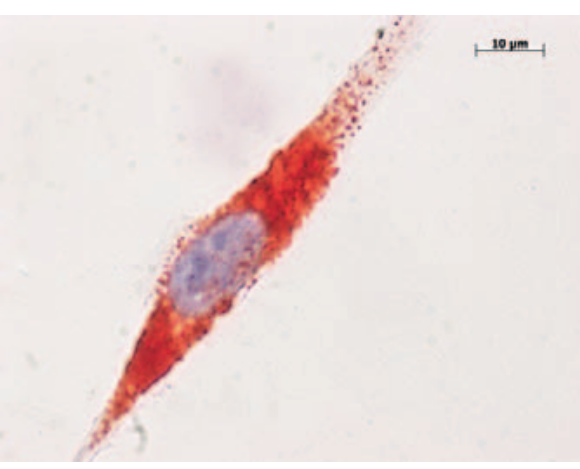

Fig. 1. Detection of collagen type I on a human primary fibroblast. Immunhistochemical staining of collagen type I indicates the distribution of the ECM protein on the cell surface. Collagen type I is stained red, cell nucleus in blue.

followed in different graduations. The simplest one is the introduction of a biomaterial which has the same physical and mechanical properties as the surrounding tissue. The body's own cells will populate the material, degrade it and thereby augment the impaired tissue. Obtaining authorization for the clinical use of acellular materials is not a major problem. For this reason most industrial companies prefer to develop biocompatible and degradable biomaterials for tissue integration.

In more complex situations where the defects are too large or too difficult to regenerate, signaling factors have to be integrated into the scaffold in order to stimulate the tissue regeneration in vivo. Products like InductOS ${ }^{\mathrm{TM}}$ (Medtronic Inc., United States) and Osigraft ${ }^{\circledR}$ (Stryker, United States) are based on a matrix in combination with bone morphogenetic factors such as BMP-2 or BMP-7. These molecules are able to induce the differentiation of precursor cells to osteoblasts, building up the newly formed bone.

The latest and most promising efforts have been undertaken by using the organism itself as a bioreactor system. Dvir et al. ${ }^{[6]}$ performed a pre-vascularization of engineered cardiadic patch in the omentum for seven days before transplantation into rat hearts.

Our research group of the Zurich University of Applied Sciences follows cellbased as well as acellular strategies for tissue augmentation and replacement. Several projects mostly supported by CTI are running. Some of them revolve around cell-based therapy for intervertebral disc degeneration and for liver regeneration. Additional tissue engineering projects focus on the development of biomaterials, e.g. soft tissue and bone substitutes.

\section{Development of Biomimetic Materials}

Biomaterials in tissue engineering applications are used to support and guide the growth of cells in specific two- or three-dimensional (2D or $3 \mathrm{D})$ structures. They take over the task of the ECM, which naturally provides cells with a supportive framework of proteins, carbohydrates and signalling molecules. The ideal biomaterial should mimic the ECM as closely as possible and reveal similar physical properties. Moreover, it should be degradable, safe and easily available at acceptable cost. Interdisciplinary cooperation is the secret to success, combining various aspects of medicine, material science, engineering and biology.

One of our recently completed projects resulted in the successful development of a natural substitute material for soft tissue restoration in the oral cavity. Especially in dental medicine, the augmentation and build-up of hard and soft tissue is an imperative issue for implant integration and functional feasibility.

Soft tissue management in the oral cavity has become one of the major challenges in cranio-maxillofacial surgery. In order to improve both functional and esthetical outcomes, autogenous soft tissue grafts, such as the free gingival graft (FGG) or the subepithelial connective tissue grafts (SCTG), are still considered to be the gold standard and are broadly used for various indications. ${ }^{[7]}$ The development of biomaterials intended to augment soft tissue volume in the oral cavity is challenging. The efficacy testing of different prototypes requires sophisticated and expensive animal models and numbers, but control over the mechanical forces is still lacking. Consequently, the transfer of animal data to human beings may be difficult. Gingival cells of the connective tissue are exposed to various and complex mechanical stresses during mastication, speech, and orthodontic movement. Especially during the procedure of wound healing, internal and external forces arise, creating pressure upon the newly formed tissue. A graft substitute has to withstand these forces in order to augment gingival soft tissue and must face the gold standard, which is the autogenous tissue graft. ${ }^{[8]}$

In our study a collagen sponge, which is under development by the dental device company Geistlich Pharma AG was investigated. ${ }^{[9]}$ Although collagen materials are commonly used as implantable materials in different fields in periodontology and implantology, ${ }^{[10]}$ low mechanical strength and fast degradation are unlikely to withstand the mechanical forces that are applied during soft tissue volume augmentation. The mechanical stability of collagen sponges can be improved by stiffening the matrix body, but increased stiffening may compromise tissue integration. ${ }^{[11]}$ Therefore the right balance between mechanical stability and uneventful soft tissue healing needs to be attained. To evaluate this balance, an in vitro testing system was established, able to mimic the mechanical and biological environment in a healing wound.

The bioreactor system was developed in our research group in cooperation with the Institute for Biomechanics of our university. It is able to apply pressure and shear forces as well as medium perfusion to specimens (Fig. 2) and therefore mimics the mechanical stresses of the human in vivo situation. Known characteristics of gingival connective tissue regeneration were incorporated in the development of the bioreactor. ${ }^{[9]}$

The results of the performed study indicate that static conditions are not suitable for investigating the natural interaction between cells and a biomedical device intended to be used for soft tissue grafting. Only mechanically stressed specimens displayed tissue specific characteristics like ECM assembly and expression of tissue remodeling signals. Moreover, cell orientation of gingival fibroblasts differs between samples cultivated under static compared to dynamic cultivated ones (Fig. 3 ). Cells are more compact and fill out the pores of the material while the cells grown on the biomaterial under static conditions are more randomly distributed and stay attached to the fibers. ${ }^{[9]}$

\section{Bioactive Factors Modulate Cell Responses}

Not only biomaterials are able to guide tissue formation, also soluble factors can modulate remodeling processes. To investigate fibroblast behavior during 3D incubation with different bioactive factors, an activity-determination assay was established in our group. By using this assay, the impact of platelet derived growth factor was investigated concerning the ability to enhance fibroblast contraction potential. It was shown in a 3D collagen gel that the growth factor alone can stimulate gel contraction similar to the serum control (Fig. 4). Together with other results this led to the conclusion that the platelet-derived growth factor is able to enhance tissue regeneration and remodeling processes. Even comparative studies with factors from different suppliers can be performed and statistically significant differences occur.

\section{Cell-based Therapies}

A crucial point for the repair and restoration of a tissue using tissue engineering techniques is that the biological substitute has to perform the appropriate physiologic/metabolic duties. Hence, the best approach seems to be represented by the 


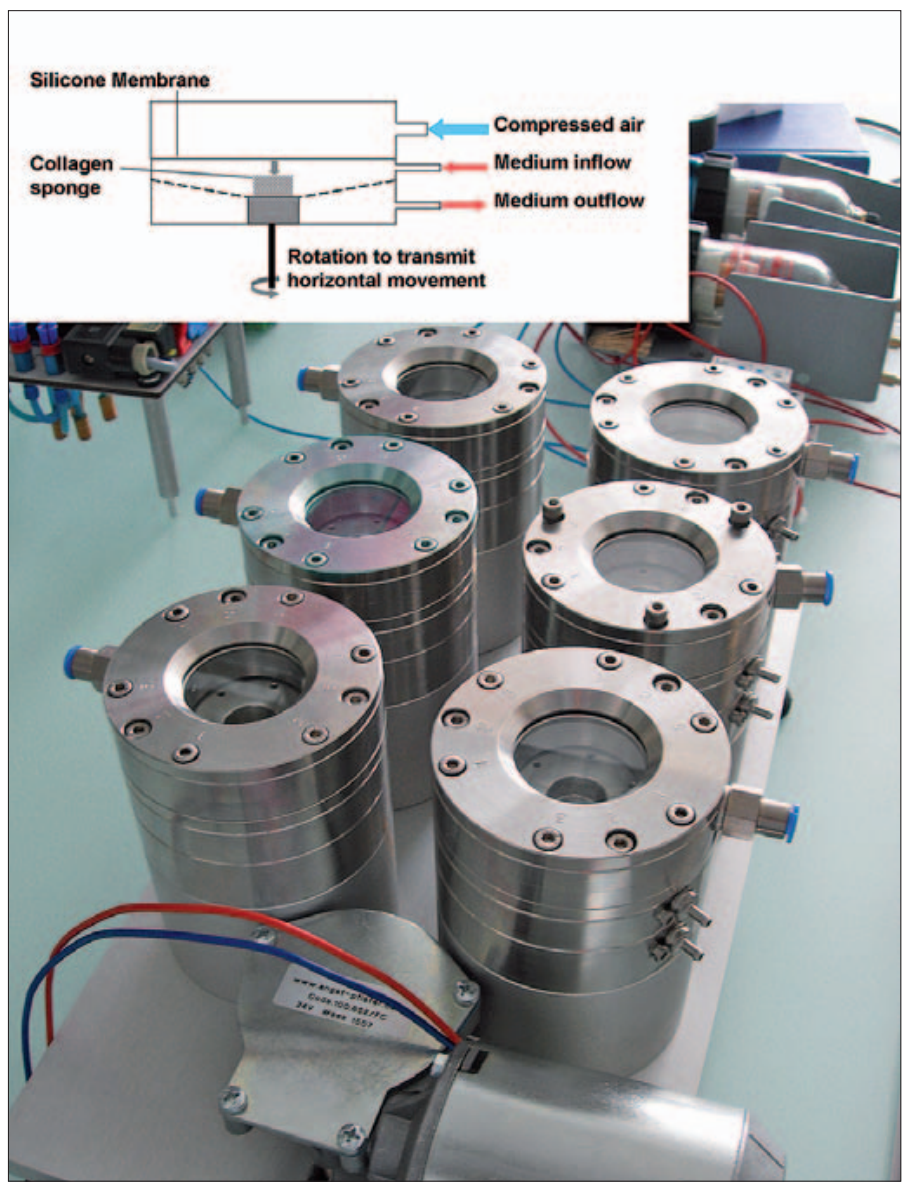

Fig. 2. Bioreactor setup with six reaction chambers made of stainless steel and a motor for horizontal movement in the core of the chamber. Pressure forces are applied via compressed air on a silicone membrane. Biomaterial e.g. a collagen sponge seeded with human cells can be placed in the centre of each chamber.
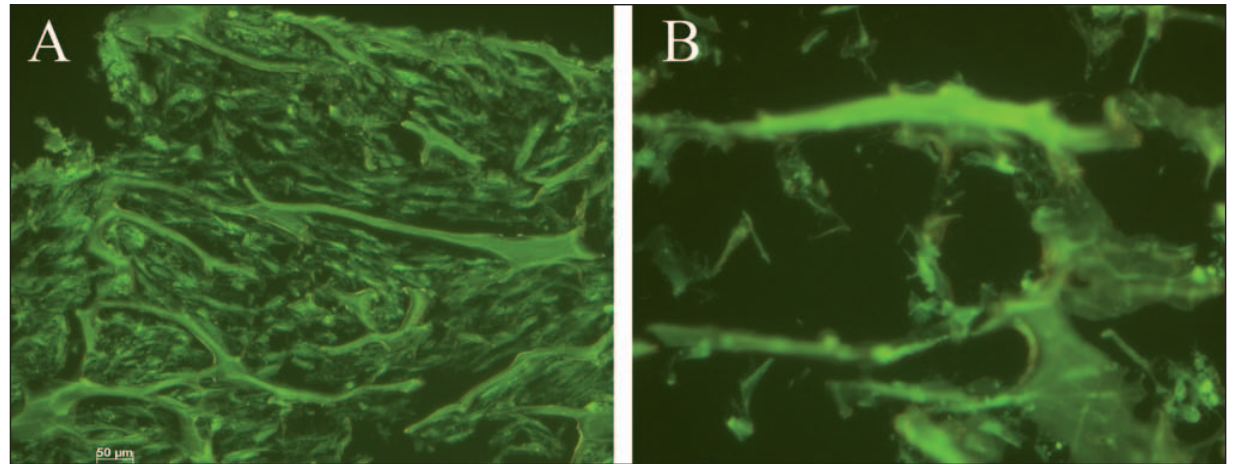

Fig. 3. Cultivation condition essentially modulates cellular growth on a biomaterial. Human primary fibroblasts were grown under (A) dynamic conditions in a bioreactor and (B) under static conditions in a cell culture plate on a collagen sponge material. Green fluorescence indicates vital cells.

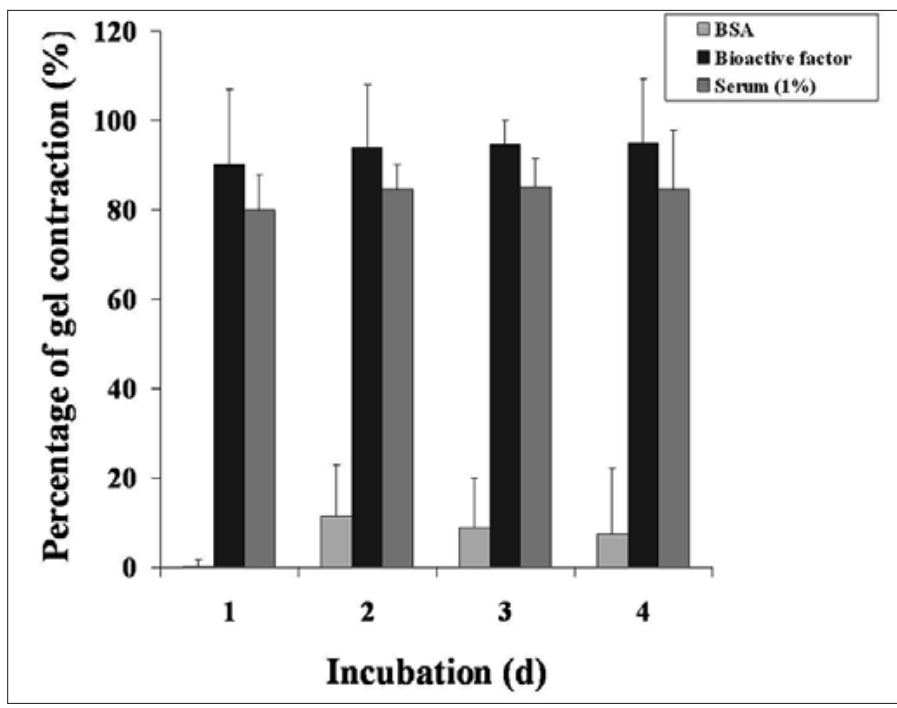

use of autologous cells that avoid inflammatory reactions in the patient and carry out the biological tissue-specific program. Cell therapies currently being investigated include the use of stem cells, cells isolated from the tissue or organ that has to be repaired, and established cell lines or cells derived from modified animals. Several pre-clinical models as well as clinical applications include chondrocytes for cartilage repair, ${ }^{[12]}$ keratinocytes and/or dermal fibroblasts for burn and wound repair, ${ }^{[13]}$ myocytes for myocardial repair, ${ }^{[14]}$ and Schwann cell transplantation to restore myelin in CNS lesions. ${ }^{[15]}$

Recently in our laboratory we established a new approach for the regeneration of the intervertebral disc (IVD), based on the 3D culture of autologous human disc cells into a polyurethane (PU)-fibrin composite for the regeneration of the nucleus pulposus (NP) tissue at an early-stage of degeneration. ${ }^{[16]}$ In our system the IVD cells were seeded on a macroporous scaffold, such as PU particles (kindly provided by polyMaterials AG, Germany), and later encapsulated into an injectable hydrogel, the fibrin glue Tissucol (Baxter, Switzerland). Several studies performed on structures composed from cells embedded in hydrogels revealed the correct ECM formation, but did not supply the required initial stability to the implant. The combination of the PU spheroids and its embedding in the fibrin glue was realized in order to provide the composite with the requested mechanical resistance and to recreate in vitro a $3 \mathrm{D}$ environment for cell differentiation. The purpose was to develop a composite on which the IVD cells could initially adhere, proliferate and have the time, after the implantation, to synthesize the ECM proteins in order to re-establish the original IVD conditions. ${ }^{[16]}$

Looked at in detail, the human primary IVD cells, fresh isolated from biopsies, were 2D expanded, seeded on PU spheroids, let to adhere (cell adhesion efficiency: $78 \pm 9.6 \%$ ) and proliferate for few days. The cell-seeded particles were then encapsulated into fibrin and the cell distribution was analyzed up to 14 days, using 4'-6-diamidino-2-phenylindole (DAPI) staining (Fig. 5C-F). During the culture period observed, the cells were able to proliferate and to migrate from the PU spheroids to the fibrin. At day 14 IVD cells reached a homogenous distribution on the glue (Fig. 5E, F) as the cells directly mixed in the fibrin and cultivated under the same conditions as PU-fibrin composites for two weeks (Fig. 5G, H). The IVD cells, migrating from the PU particles to the fibrin, switched from a macroporous (a 2D situation for the cell size) to a microporous environment, simulating 3D in vivo conditions. The cell proliferation, ob- 

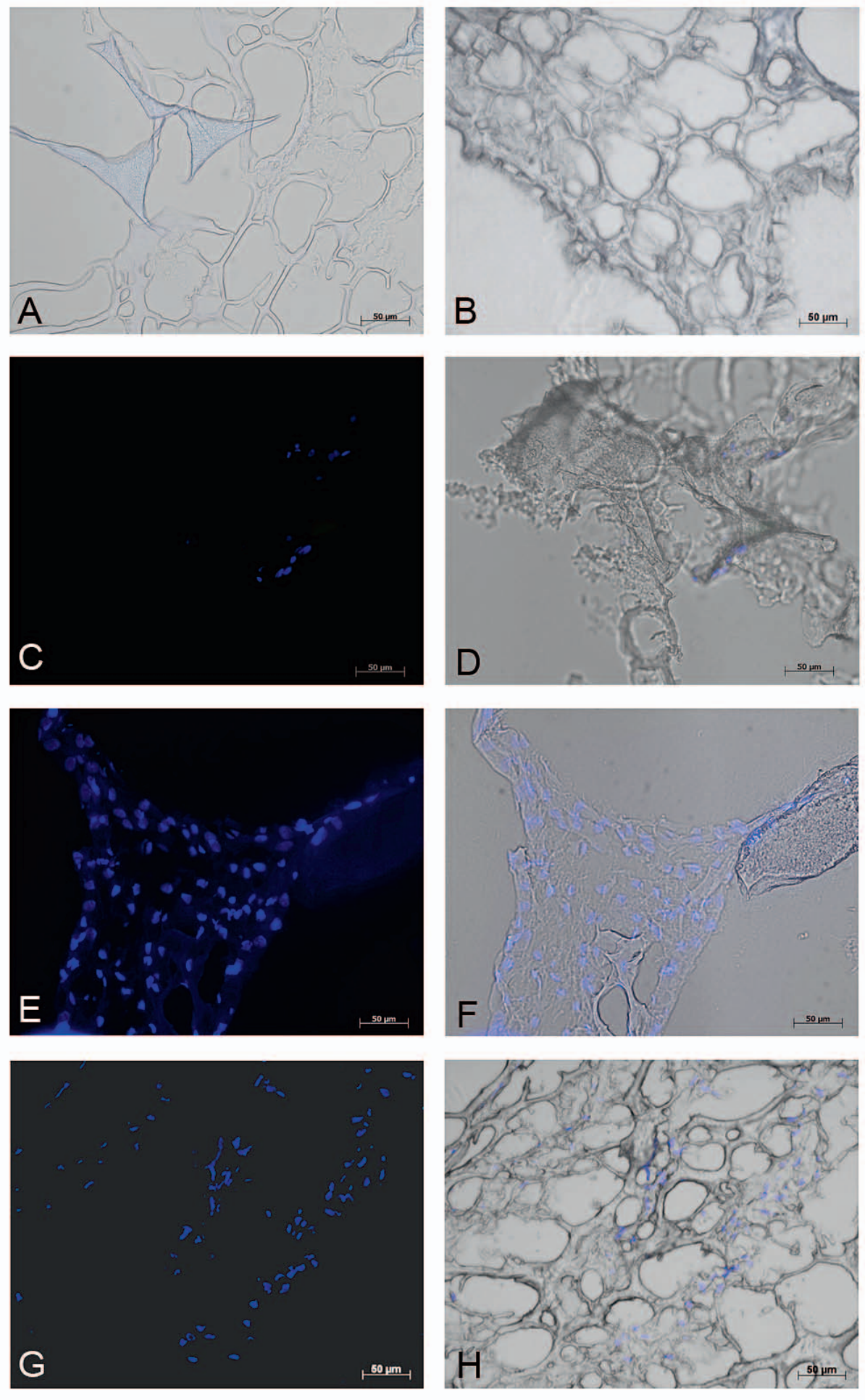

Fig. 5. DAPI stained cryosections of PU-fibrin composites and fibrin structures. Blanks: (A) PU-fibrin and (B) fibrin matrix without cells. Cell expansion and distribution into the PU-fibrin structure after $3 d(C)$, and $14 d(E)$ observed by fluorescence microscopy, and (D, F) overlay with light microscopy. (G) Cell distribution into the fibrin after $14 \mathrm{~d}$ culture observed by fluorescence microscopy and $(\mathrm{H})$ overlay with light microscopy (20x magnification).

served with DAPI staining, was confirmed from the DNA content quantification (Fig. 6A). The DNA amount increased throughout the culture, reaching a DNA value of $3.27 \pm 0.86 \mu \mathrm{g}$ after $7 \mathrm{~d}$ (2-fold vs. $3 \mathrm{~d}$ cultures) and of $3.58 \pm 0.79 \mu \mathrm{g}$ after $14 \mathrm{~d}$ (Fig. 6A). Moreover, considering the im- portance of the ECM in a healthy disc, the expression of collagen and glycosamminoglycans (GAG) was analyzed. The collagen showed a significant increase in the second week of culture (Fig. 6B), while a constant GAG amount was detected over the period analyzed (Fig. 6C). The pheno- typic characterization analyses (gene expression studies) confirmed the differentiation of the IVD cells onto the PU-fibrin composites in vitro. ${ }^{[16]}$

Our experiments demonstrated the capability of human IVD cells to grow, express ECM proteins and re-differentiate onto PU-fibrin structures, suggesting the suitability of our model for disc regeneration.

\section{Automation - A Key Prerequisite for Clinical Application}

It is in the nature of biological products that they vary. Primary cell isolation and cultivation involve many experiencebased steps such as surgical tissue collection, enzymatic digestion of the tissue, cell culture and analysis. In addition, the variation among patients' biopsies impedes the standardization of the procedure and therefore experienced experts are necessary. ${ }^{[17]}$ To ensure an effective, reproducible and affordable production process and to overcome, or at least minimize, the hurdles in the approval process, a standardized and automated procedure to deliver cells and tissues is crucial.

In a recently completed project we realized an automated tissue and cell processing platform based on the Liquid Handling Robot Freedom EVO ${ }^{\circledR} 150$ (Tecan ${ }^{\circledR}$, Switzerland) (Fig. 7). We were able to isolate and to proliferate human primary intervertebral disc cells in a fully automated way. We could clearly show that the automated process was at least as good as the manual process or even better. In situ confluence measurement, giving information on cell growth, allowed automated process control. Furthermore, the differentiation of the cells was determined by immunofluorescent staining of specific markers automatically analyzed with an integrated microscope. The results have been submitted for publication in Journal of the Association for Laboratory Automation.

The integrated quality control and process decision permits the standardization of a complex tissue engineering process. The innovation of this robot is a key prerequisite for future clinical application of tissue engineering. This automation is a major leap forward in bringing tissue engineering technologies from the bench to the bedside.

\section{Conclusion: "The Future of Medicine is Regenerative"}

Regenerative medicine is one of the great hopes of the future for the treatment of organ damage, cancer and degenerative diseases. Our institute at the Zurich University of Applied Sciences and Biotechnet 


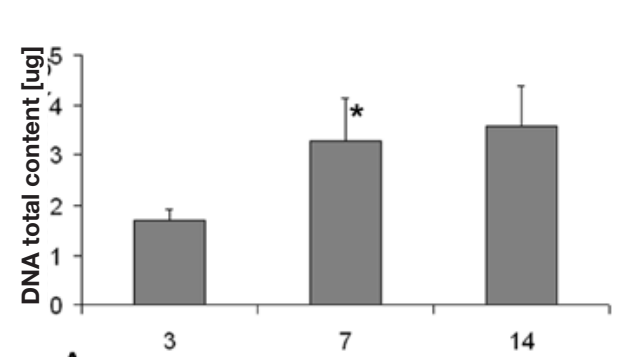

A Cultivation time [d]

14

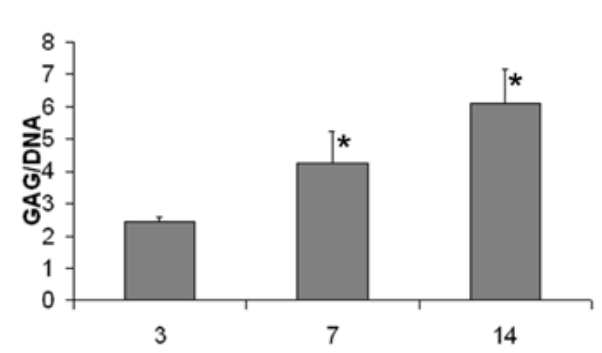

C Cultivation time [d]

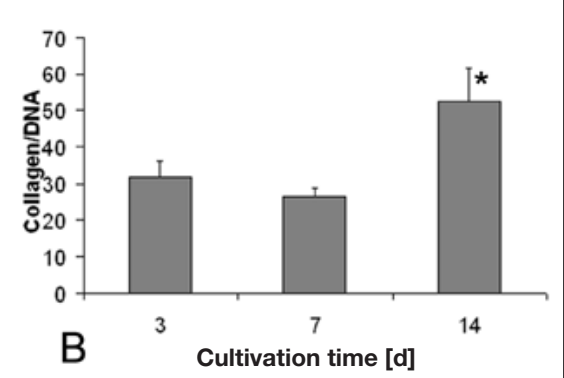

[12] a) L. J. Micheli, J. B. Moseley, A. F. Anderson, J. E. Browne, C. Erggelet, R. Arciero, F. H. Fu, B.R. Mandelbaum, J. Pediatr. Orthop. 2006, 26, 455; b) L. Peterson, T. Minas, M. Brittberg, A. Lindahl, J. Bone Joint Surg. Am. 2003, 85, 17; c) M. Brittberg, Am. J. Sports Med. 2010, 38, 1259.

[13] a) G. Travia, P. A. Palmisano, V. Cervelli, G. Esposito, C. U. Casciani, Annals of Burns and Fire Disasters 2003, XVI, 1; b) D. J. Geer, D. D. Swartz, S. T. Andreadis, Am. J. Pathol. 2005, 167, 1575.

[14] a) M. S. Penn, G. S. Francis, S. G. Ellis, J. B. Young, P. M. McCarthy, E. J. Topol, Prog. Cardiovasc. Dis. 2002, 45, 21; b) N. Tran, Y. Li, S. Bertrand, S. Bangratz, J. P. Carteaux, J. F. Stoltz, J. P. Villemot, Biorheology 2003, 40, 411.

[15] B. Cheng, Z. Chen, Microsurgery 2002, 22, 133.

[16] C. Mauth, E. Bono, S. Haas, G. Paesold, H. Wiese, G. Maier, N. Boos, U. Graf-Hausner, Eur. Cell Mater. 2009, 18, 27.

[17] R. Kato, D. Iejima, H. Agata, I. Asahina, K. Okada, M. Ueda, H. Honda, H. Kagami, Tissue Eng. Part C Methods 2010, 16, 947.

Fig. 6. Biochemical analyses of DNA content (A), collagen (B) and GAG (C) to DNA ratio within PUfibrin structures after 3,7 and $14 \mathrm{~d}$ culture. The collagen/DNA ratio as well as the GAG/DNA ratio was determined by dividing the ECM component $(\mu \mathrm{g})$ by DNA $(\mu \mathrm{g})$ per each PU-fibrin structure. *, $p<0.05$ compared cultures of 7 versus $3 d$, and 14 versus $7 d$ in culture, respectively.

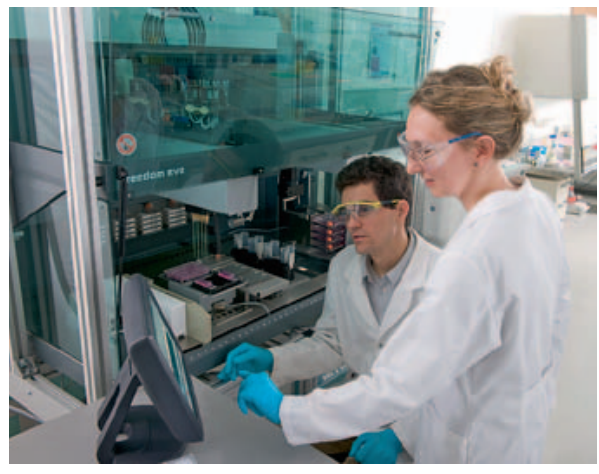

Fig. 7. The developed instrument for automated tissue processing. Once the scripts to be executed are defined, the instrument works without need of human intervention.

of Switzerland, the national consortium of biotechnology, contribute to the exciting development of these technologies by their activities in research, development and the education of students.

Painting too bright a picture of the potential of tissue engineering will probably not help the development and the creation of confidence in this innovative technology. To fulfill the promise of tissue engineering, especially to construct in vitro human organs, several challenges relating to scientific, technological and also society issues need to be met. However, in the medium to long term, the manifold research activities will result in the development of novel products to fight diseases for which no treatment is available today.

\section{Acknowledgments}

We thank our collaboration partners of the Spine Research Group, University of Zurich, and our industrial partners Geistlich Pharma AG, Tecan Schweiz AG, polyMaterials AG, and CTI for funding.

Received: September 6, 2010

[1] R. Langer, J. P. Vacanti, Science 1993, 260, 920.

[2] M. J. Jayo, D. Jain, J. W. Ludlow, R. Payne, B. J. Wagner, G. McLorie, T. A. Bertram, Regen. Med. 2008, 3, 671.

[3] a) J. S. Hammond, I. J. Beckingham, K. M. Shakesheff, Expert Rev. Med. Devices 2006, 3 , 21; b) M. Wang, H. Pei, L. Zhang, L. Guan, R. Zhang, Y. Jia, B. Li, W. Yue, Y. Wang, X. Pei, Tissue Eng. Part C Methods 2010, 24, 1.

[4] H. Jawad, A. R. Lyon, S. E. Harding, N. N. Ali, A. R. Boccaccini, Br. Med. Bull. 2008, 87, 31.

[5] A. K. Bock, D. Ibarreta, E. Rodriguez-Cerezo, EUR 21000 EN, 2003

[6] T. Dvir, A. Kedem, E. Ruvinov, O. Levy, I. Freeman, N. Landa, R. Holbova, M. S. Feinberg, S. Dror, Y. Etzion, J. Leor, S. Cohen, PNAS 2009, 106, 14990.

[7] a) R. E. Jung, D. W. Siegenthaler, C. H. Hämmerle, Int. J. Periodontics Restorative Dent. 2004, 24, 545; b) F. Cairo, U. Pagliaro, M. Nieri, J. Clin. Periodontol. 2008, 35, 136; c) D. S. Thoma, G. L. Benić, M. Zwahlen, C. H. Hämmerle, R. E. Jung, Clin. Oral Implants Res. 2009, 20, 146

[8] S. Studer, C. Lehner, A. Bucher, P. Schärer, J. Prosthet. Dent. 2000, 83, 402.

[9] S.H. Mathes, L. Wohlwend, L. Uebersax, R. V. Mentlen, D. S. Thoma, R. E. Jung, C. Görlach, U. Graf-Hausner, Biotechnol. Bioeng. 2010, 107, 1033.

[10] a) J. Behring, R. Junker, X. F. Walboomers, B. Chessnut, J. A. Jansen, Odontology 2008, 96, 1; b) B. C. Jham, N. G. Nikitakis, M. A. Scheper, J. C. Papadimitriou, B. A. Levy, H. Rivera, J. Oral Maxillofac. Surg. 2009, 67, 280.

[11] a) D. Rothamel, F. Schwarz, M. Sager, M. Herten, A. Sculean, J. Becker, Clin. Oral. Implants Res. 2005, 16, 369; b) F. Schwarz, D. Rothamel, M. Herten, M. Sager, J. Becker, Clin. Oral Implants Res. 2006, 17, 403. 